\title{
Interactions between selected bile salts and Triton X-100 or sodium lauryl ether sulfate
}

\author{
Dejan M Ćirin", Mihalj M Poša and Veljko S Krstonošić
}

\begin{abstract}
Background: In order to develop colloidal drug carriers with desired properties, it is important to determine physico-chemical characteristics of these systems. Bile salt mixed micelles are extensively studied as novel drug delivery systems. The objective of the present investigation is to develop and characterize mixed micelles of nonionic (Triton X-100) or anionic (sodium lauryl ether sulfate) surfactant having oxyethylene groups in the polar head and following bile salts: cholate, deoxycholate and 7-oxodeoxycholate.

Results: The micellization behaviour of binary anionic-nonionic and anionic-anionic surfactant mixtures was investigated by conductivity and surface tension measurements. The results of the study have been analyzed using Clint's, Rubingh's, and Motomura's theories for mixed binary systems. The negative values of the interaction parameter indicate synergism between micelle building units. It was noticed that Triton X-100 and sodium lauryl ether sulfate generate the weakest synergistic interactions with sodium deoxycholate, while 7-oxodeoxycholate creates the strongest attractive interaction with investigated co-surfactants.
\end{abstract}

Conclusion: It was concluded that increased synergistic interactions can be attributed to the larger number of hydrophilic groups at $\alpha$ side of the bile salts. Additionally, 7-oxo group of 7-oxodeoxycholate enhance attractive interactions with selected co-surfactants more than 7-hydroxyl group of sodium cholate.

\section{Background}

Bile salts are steroid compounds with planar amphiphilic properties [1]. These surfactant characteristics result from the combination of the polar hydroxyl groups on the concave $\alpha$-face and the methyl groups on the convex $\beta$-face $[1,2]$. Bile salts in aqueous solution form aggregates mainly due to hydrophobic attractive interactions of the apolar $\beta$-faces [3].

However, further stabilisation and aggregation occurs through intermolecular hydrogen bonds formation $[4,5]$. It is known that bile salts form micelles at concentrations above the critical micelle concentration $(\mathrm{cmc})$ $[3,6,7]$. The more hydrophobic bile salt is, the lower critical micelle concentration it has, due to more stable hydrophobic aggregations [6,7].

Bile salts are known as permeability enhancers to increase drug penetration through various biological membranes by interacting with phospholipids in cell membranes $[8,9]$. Micelles of bile salts have been

\footnotetext{
* Correspondence: dejan.cirin@gmail.com

Department of Pharmacy, Faculty of Medicine, University of Novi Sad, Hajduk Veljkova 3, 21000 Novi Sad, Serbia
}

investigated in different pharmaceutical formulations where they solubilise poorly soluble drugs [10-13]. The ability of bile salts to achieve this is mainly dependent on their hydrophobicity. Bile salts with more hydrophobic micellar core have greater ability to accept hydrophobic pharmaceutical ingredients [11]. Solubilisation capacity of the micellar systems can be increased by chemical modification of the bile salt steroid skeleton. Another method to improve solubilisation properties of micellar systems is to produce mixed micelles by combining individual surfactants with different aggregation properties ( $c m c$ values) [14,15]. Furthermore, intermolecular hydrophilic interactions between polar groups in certain bile salt mixed micelles are known to greatly increase stability of these binary systems [16].

In the pharmaceutical area, various nonionic co-surfactants have been used in bile salt-nonionic binary systems due to ability of these mixtures to improve solubilisation of poorly soluble drugs [17-23]. However, in studies of bile salt-anionic binary mixtures, sodium dodecyl sulfate has been predominantly used as an anionic co-surfactant [24-27]. 
Triton X-100 (TRX) is a nonionic surfactant with a hydrophilic polyethylene oxide group (on average it has 9.5 oxyethylene groups $(\mathrm{OE})$ ) and a hydrophobic group. TRX ( $p$-(1,1,3,3-tetramethylbutyl)phenoxypolyoxyethylene glycol) differs from conventional nonionic surfactants, having hydrophilic part longer than the hydrophobic region of the molecule. Sodium lauryl ether sulfate (SLES) is an anionic surfactant with one to four hydrophilic OE groups adjacent to the anionic sulfate group. Its basic structure is $\mathrm{C} 12 \mathrm{H} 25(\mathrm{OCH} 2 \mathrm{CH} 2)$ $x$ OSO3Na where $x$ represents the number of OE groups in the chain. For this work, SLES having two OE groups was used.

To the best of our knowledge, work on physico-chemical characterisation of binary mixtures consisting of bile salt and polyoxyethylene type of co-surfactant has been done only with individual bile salts $[17,28,29]$. However, there is no comparative data on the physicochemical properties of mixed micelles of TRX or SLES with different bile salts. According to the authors' knowledge investigations of SLES-bile salt binary systems have not been reported previously.

In the present work, we have tested a set of three bile salts (sodium cholate $(C)$, sodium deoxycholate (D) or sodium 7-oxodeoxycholate (OD)) to interact with TRX or SLES. The results of the study have been analysed using Clint's, Rubingh's, and Motomura's theories for mixed binary systems [30-32].

The first aim of this study was to investigate how different combinations of hydroxyl and oxo groups on the concave $\alpha$ - face of the bile salt skeleton can influence the interactions with anionic and nonionic cosurfactant having polar OE groups in the hydrophilic region. The second goal was to evaluate how structural differences in selected anionic and nonionic co-surfactant impact micelization behaviour of developed mixed micelles.

It is envisioned that insight on the formation of the micellar structures obtained in this study could be used to develop drug delivery formulations with optimized physico-chemical properties.

\section{Results and Discussion}

\subsection{Determination of the $\mathrm{cmc}$ values of individual} surfactants

The $c m c$ value of TRX was obtained by surface tension measurements. The $c m c$ values of individual bile salts (D, C, and OD) and SLES were acquired through conductivity and surface tension studies. The experimentally obtained $c m c$ values, with standard deviations of three determinations, for individual surfactants $\left(c m c^{e x}\right)$ are presented in Table 1 and compared to the literature values $\left(c m c^{l i t}\right)$ [33-35]. The $c m c$ value for OD has not been found in literature.
Table 1 Experimentally obtained critical micelle concentrations of the individual surfactants compared to the literature values

\begin{tabular}{cccc}
\hline Surfactant & \multicolumn{2}{c}{$\boldsymbol{c m}^{\boldsymbol{e x}}(\mathbf{m M})$} & \multirow{2}{*}{$\boldsymbol{c}$} \\
\cline { 2 - 3 } & $\boldsymbol{a}$ & $\boldsymbol{b}$ & \\
\hline D & $4.30 \pm 0.07$ & $4.25 \pm 0.05$ & 4.16 \\
C & $12.90 \pm 0.16$ & $12.78 \pm 0.14$ & 12.8 \\
OD & $61.10 \pm 0.9$ & $59.50 \pm 1.2$ & - \\
SLES & $0.80 \pm 0.01$ & $0.82 \pm 0.02$ & 0.8 \\
TRX & - & $0.240 \pm 0.004$ & 0.24
\end{tabular}

$a$ the values obtained from conductivity measurements.

$b$ the values obtained from surface tension measurements.

$c m c^{e x}$ the mean values \pm standard deviation of three determinations

$\mathrm{cmc}^{\text {lit }}$ the $\mathrm{cmc}$ values obtained from the literature

\subsection{Determination of the $\mathrm{cmc}$ values of the binary mixtures}

The critical micelle concentrations of the binary mixtures were studied using conductivity and surface tension measurements, at different mole fractions of TRX or SLES $(\alpha)$. Prepared mixtures consisted of $0.1,0.2,0.3$, 0.4 or 0.5 mole fractions of TRX or SLES $(\alpha)$. For the conductivity studies, a break in the conductivity (specific conductivity, $\kappa$ ) versus concentration plot indicated the onset of the micellization process, since this break is characteristic of micelle formation and represents the $c m c$ (Figure 1). The $c m c$ values obtained in the conductivity measurements were acquired from the intersection of the straight lines created above and below the $c m c$. The straight line above the $c m c$ value was determined by linear regression starting at the highest concentration, while the straight line below the $c m c$ value was obtained by linear regression beginning at the lowest concentration of investigated binary mixtures. The regression diagnostic was constantly performed after

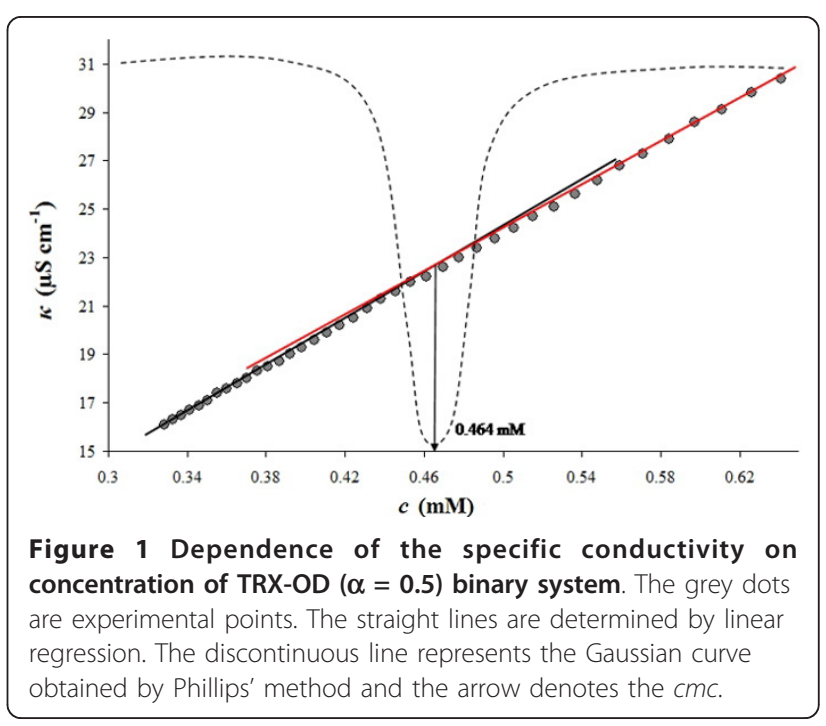


addition of each subsequent experimental point. If an experimental point was identified as an outlier, by measuring Cook's distance [36], the regression would be stopped and the straight line would be formed using previously added experimental points (Figure 1).

Figure 1 shows the intersection of the regression lines. However, this method for the determination of $\mathrm{cmc}$ values is reliable only if there is an abrupt change in conductivity. As seen in Figure 1, the change in conductivity does not show an abrupt alteration and the intersection point is not clearly detectable. Since the uncertainty in determination of $c m c$ values is increased, Phillips' method [37] was applied in order to obtain the $c m c$ values more reliably. In this approach, the $c m c$ is considered as the concentration at which the maximum change in a physical property of the solution, $\varphi$, versus concentration curve occurs.

$$
\left(\mathrm{d}^{3} \phi / \mathrm{dc}^{3}\right) \mathrm{c}=0 ; \mathrm{c}=c m c
$$

Phillips' method was employed as proposed by Mosquera et al [38]. The Runge-Kutta integration was applied and a least squares fitting method, according to Levenberg-Maquardt, was used. The usage of Phillips' method for determination of $c m c$ values has been reported in previous studies, as well $[39,40]$. Figure 1 shows the application of Phillips' method.

In order to validate the results determined by means of conductivity measurements, the $\mathrm{cmc}$ values were remeasured using surface-tension studies. Plots of surface tension $(\gamma)$ versus concentration of binary system were obtained, where a break point corresponds to the cmc. Figure 2 shows the $c m c$.

In order to evaluate reproducibility of used methods, all conductivity and surface tension measurements were repeated three times. The $c m c$ determination error did not exceed 3\%. Extended t-test $(\mathrm{p}<0.05)$ shows that the mean $c m c$ values determined using conductivity and

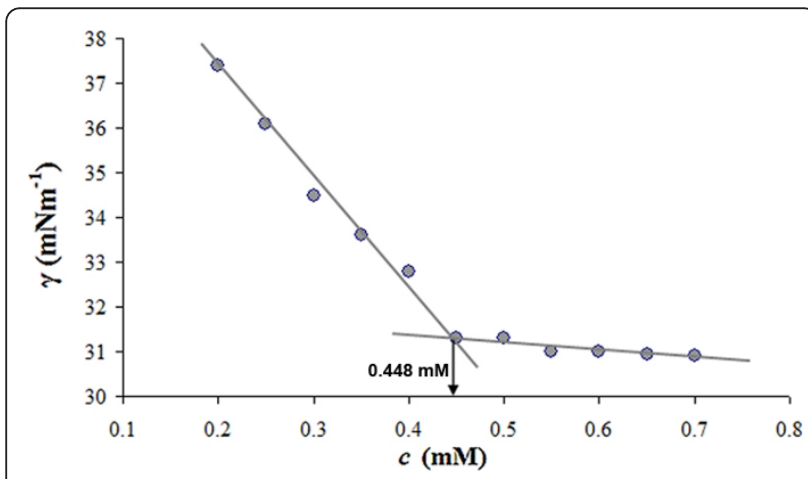

Figure 2 Dependence of surface tension on concentration of TRX-OD $(\alpha=0.5)$ binary system. The arrow denotes the $\mathrm{cmc}$. surface tension measurements belong to the same population.

The experimentally obtained $c m c$ values, with standard deviations of three determinations, for the TRX-bile salt and SLES-bile salt binary mixtures are presented in Tables 2 and 3 respectively.

As seen in Tables 2 and 3 the $c m c$ values obtained in conductivity and surface tension studies are in good agreement. Since there is no statistically significant difference between the $c m c$ values determined by means of the two methods, the $c m c$ values acquired by conductivity measurements were used in further calculations.

\subsection{Determination of the physico-chemical parameters}

In order to study the influence of the structure of bile salts on the formation of mixed micelles with TRX or SLES, the physico-chemical parameters of the micellar systems were calculated using experimentally obtained $c m c$ values. The determined physico-chemical parameters are: the critical micelle concentration of ideal mixtures $\left(c m c^{i d}\right)$, the mole fraction of the more hydrophobic surfactant in the ideal mixed micelle $\left(x^{i d}\right)$, the mole fraction of the more hydrophobic surfactant in the real mixed micelle $\left(x_{1}\right)$ and the $\beta$ parameter.

Table 2 Comparision of the experimentally obtained critical micelle concentrations of investigated TRX-bile salt binary systems

\begin{tabular}{|c|c|c|}
\hline \multirow[t]{2}{*}{$\alpha[$ TRX $]$} & \multicolumn{2}{|c|}{$c m c^{e x}(m M)$} \\
\hline & $a$ & $b$ \\
\hline & \multicolumn{2}{|c|}{ D } \\
\hline 0 & $4.30 \pm 0.07$ & $4.20 \pm 0.05$ \\
\hline 0.1 & $1.545 \pm 0.023$ & $1.533 \pm 0.021$ \\
\hline 0.2 & $0.832 \pm 0.017$ & $0.821 \pm 0.019$ \\
\hline 0.3 & $0.617 \pm 0.015$ & $0.605 \pm 0.013$ \\
\hline 0.4 & $0.498 \pm 0.012$ & $0.491 \pm 0.011$ \\
\hline \multirow[t]{2}{*}{0.5} & $0.416 \pm 0.012$ & $0.420 \pm 0.010$ \\
\hline & \multicolumn{2}{|c|}{ C } \\
\hline 0 & $12.90 \pm 0.16$ & $12.78 \pm 0.14$ \\
\hline 0.1 & $1.565 \pm 0.020$ & $1.579 \pm 0.018$ \\
\hline 0.2 & $0.848 \pm 0.019$ & $0.855 \pm 0.018$ \\
\hline 0.3 & $0.633 \pm 0.014$ & $0.619 \pm 0.012$ \\
\hline 0.4 & $0.510 \pm 0.013$ & $0.501 \pm 0.010$ \\
\hline \multirow[t]{2}{*}{0.5} & $0.423 \pm 0.011$ & $0.410 \pm 0.09$ \\
\hline & \multicolumn{2}{|c|}{ OD } \\
\hline 0 & $61.10 \pm 0.20$ & $59.50 \pm 0.25$ \\
\hline 0.1 & $2.021 \pm 0.029$ & $2.035 \pm 0.025$ \\
\hline 0.2 & $1.040 \pm 0.020$ & $1.028 \pm 0.022$ \\
\hline 0.3 & $0.723 \pm 0.017$ & $0.711 \pm 0.016$ \\
\hline 0.4 & $0.560 \pm 0.013$ & $0.551 \pm 0.011$ \\
\hline 0.5 & $0.464 \pm 0.011$ & $0.448 \pm 0.010$ \\
\hline
\end{tabular}

$a$ the values obtained from conductivity measurements.

$b$ the values obtained from surface tension measurements.

$c m c^{e x}$ the mean values \pm standard deviation of three determinations 
Table 3 Comparision of the experimentally obtained critical micelle concentrations of investigated SLES-bile salt binary systems

\begin{tabular}{|c|c|c|}
\hline \multirow[t]{2}{*}{$\alpha[$ SLES $]$} & \multicolumn{2}{|c|}{$c m c^{e x}(\mathrm{mM})$} \\
\hline & $A$ & $b$ \\
\hline & \multicolumn{2}{|c|}{$\mathrm{D}$} \\
\hline 0 & $4.30 \pm 0.07$ & $4.20 \pm 0.05$ \\
\hline 0.1 & $2.857 \pm 0.041$ & $2.845 \pm 0.037$ \\
\hline 0.2 & $2.203 \pm 0.035$ & $2.193 \pm 0.036$ \\
\hline 0.3 & $1.845 \pm 0.032$ & $1.841 \pm 0.034$ \\
\hline 0.4 & $1.528 \pm 0.031$ & $1.517 \pm 0.030$ \\
\hline \multirow[t]{2}{*}{0.5} & $1.322 \pm 0.029$ & $1.305 \pm 0.032$ \\
\hline & \multicolumn{2}{|c|}{$C$} \\
\hline 0 & $12.90 \pm 0.16$ & $12.78 \pm 0.14$ \\
\hline 0.1 & $4.461 \pm 0.058$ & $4.477 \pm 0.065$ \\
\hline 0.2 & $2.864 \pm 0.045$ & $2.875 \pm 0.050$ \\
\hline 0.3 & $2.165 \pm 0.039$ & $2.181 \pm 0.042$ \\
\hline 0.4 & $1.730 \pm 0.036$ & $1.725 \pm 0.043$ \\
\hline \multirow[t]{2}{*}{0.5} & $1.427 \pm 0.035$ & $1.438 \pm 0.039$ \\
\hline & \multicolumn{2}{|c|}{$\mathrm{OD}$} \\
\hline 0 & $61.10 \pm 0.20$ & $59.50 \pm 0.25$ \\
\hline 0.1 & $6.511 \pm 0.088$ & $6.395 \pm 0.081$ \\
\hline 0.2 & $3.598 \pm 0.054$ & $3.620 \pm 0.049$ \\
\hline 0.3 & $2.493 \pm 0.050$ & $2.475 \pm 0.052$ \\
\hline 0.4 & $1.906 \pm 0.041$ & $1.885 \pm 0.044$ \\
\hline 0.5 & $1.549 \pm 0.034$ & $1.529 \pm 0.038$ \\
\hline
\end{tabular}

$a$ values obtained from conductivity measurements.

$b$ values obtained from surface tension measurements.

$\mathrm{cmc}^{\mathrm{ex}}$ the mean values \pm standard deviation of three determinations

The physico-chemical parameters for the TRX-bile salt and SLES-bile salt mixed micelles were calculated according to the equations presented in Table 4 . The obtained values are presented in Tables 5 and 6 respectively.

\subsection{Discussion}

The results show deviation of the $c m c^{e x}$ values from those calculated according to Clint's theory indicating nonideal behaviour of examined mixtures and existence of mutual interactions of the components in the micelles. The difference between $x^{i d}$ and $x_{1}$ also indicates nonideal behaviour of the mixtures.

From Tables 5 and 6 , it can be observed that values of the $\beta$ parameter come out to be negative at all molar ratios of the mixtures. The more negative value of the $\beta$ interaction parameter indicates stronger synergistic interactions between the components. These values correspond to the deviation between the experimentaly obtained and calculated $c m c$ values and indicate synergism in all investigated TRX-bile salt and SLES-bile salt mixtures. In Table 7, the average values of the interaction parameter $\left(\beta_{a v}\right)$ for analysed binary systems are presented in order to make the results easily comparable.

The average interaction parameter values show that TRX generates stronger sinergistic interactions with investigated bile salts than SLES. Many recent studies have confirmed that synergistic effect generally occurs in anionic-nonionic surfactant mixtures, improving stability of the system [41-48]. In previously investigated sodium deoxycholate-Triton X-100 binary system, synergistic interaction is reported, with $\beta_{a v}=-0.9$ [28]. This value is in good agreement with the average value of the interaction parameter reported in our study $(-0.88)$. It is known that in mixed miceles composed of two anionic amphihpiles, repulsive electrostatic interactions between

Table 4 Physico-chemical parameters of mixed micelles

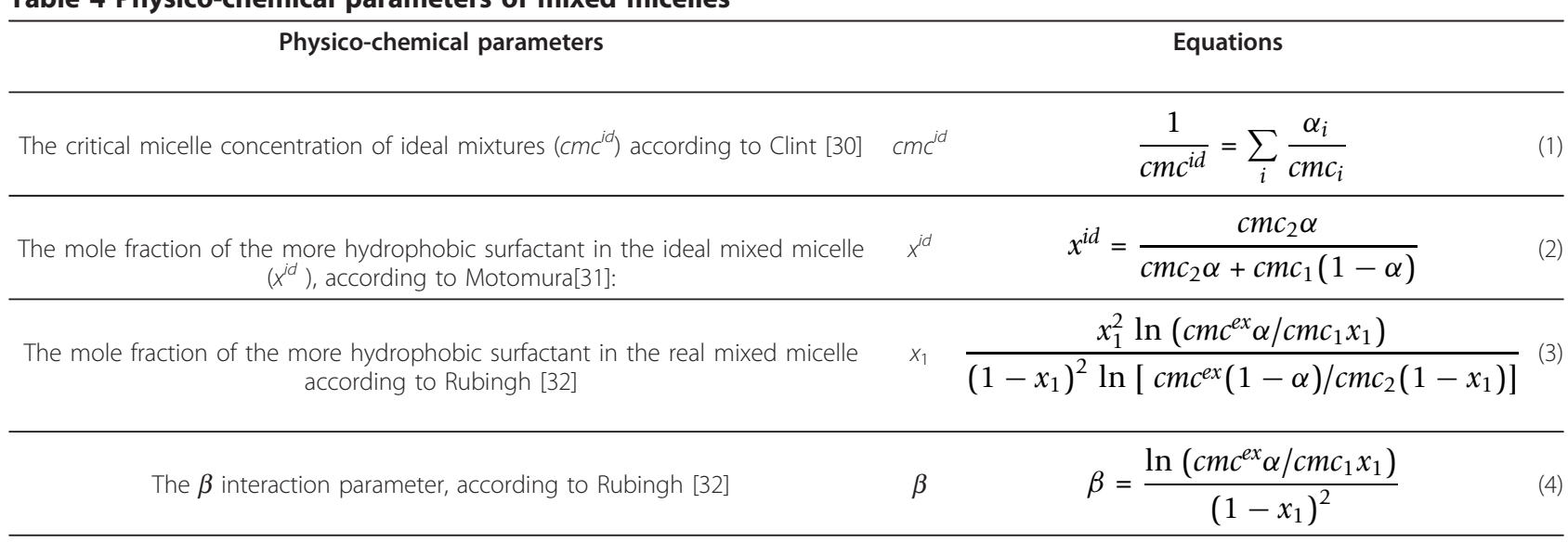

$\alpha_{i}$ the mole fraction in the solution of component $i$,

$\mathrm{cmc}_{i}$ the experimentally obtained $\mathrm{cmc}$ of component $i$.

$\mathrm{Cmc}_{1}$ the experimentally obtained $\mathrm{cmc}$ values of the more hydrophobic surfactant (TRX or SLES)

$\mathrm{Cmc}_{2}$ the $\mathrm{cmc}$ of $\mathrm{D}, \mathrm{C}$ or OD

$c m c^{e x}$ the experimentally obtained $c m c$ values of investigated binary systems

$\alpha$ the mole fraction of the more hydrophobic surfactant in the solution (TRX or SLES) 
Table 5 Comparison of experimental with ideal parameters and interaction parameter $(\beta)$ of the TRX-bile salt mixtures

\begin{tabular}{|c|c|c|c|c|c|}
\hline \multirow[t]{2}{*}{$\alpha[$ TRX $]$} & $c m c^{e x}$ & $c m c^{i d}$ & \multirow[t]{2}{*}{$x_{1}$} & \multirow[t]{2}{*}{$x^{\text {id }}$} & \multirow[t]{2}{*}{$\beta$} \\
\hline & \multicolumn{2}{|c|}{$(\mathrm{mM})$} & & & \\
\hline \multicolumn{6}{|c|}{ Sodium deoxycholate } \\
\hline 0 & 4.30 & & - & - & - \\
\hline 0.1 & 1.545 & 1.597 & 0.654 & 0.665 & -0.16 \\
\hline 0.2 & 0.832 & 0.981 & 0.739 & 0.817 & -0.97 \\
\hline 0.3 & 0.617 & 0.711 & 0.803 & 0.885 & -1.04 \\
\hline 0.4 & 0.498 & 0.554 & 0.850 & 0.923 & -1.06 \\
\hline 0.5 & 0.416 & 0.455 & 0.881 & 0.947 & -1.16 \\
\hline \multicolumn{6}{|c|}{ Sodium cholate } \\
\hline 0 & 12.90 & & - & - & - \\
\hline 0.1 & 1.565 & 2.06 & 0.733 & 0.857 & -1.64 \\
\hline 0.2 & 0.848 & 1 & 0.785 & 0.931 & -2.27 \\
\hline 0.3 & 0.633 & 0.737 & 0.838 & 0.954 & -2.19 \\
\hline 0.4 & 0.510 & 0.584 & 0.877 & 0.973 & -1.96 \\
\hline 0.5 & 0.423 & 0.471 & 0.901 & 0.982 & -2.26 \\
\hline \multicolumn{6}{|c|}{ Sodium 7-oxodeoxycholate } \\
\hline 0 & 61.10 & & & & \\
\hline 0.1 & 2.021 & 2.32 & 0.870 & 0.965 & -1.93 \\
\hline 0.2 & 1.040 & 1.18 & 0.893 & 0.984 & -2.61 \\
\hline 0.3 & 0.723 & 0.79 & 0.920 & 0.991 & -2.78 \\
\hline 0.4 & 0.560 & 0.596 & 0.942 & 0.994 & -2.75 \\
\hline 0.5 & 0.464 & 0.478 & 0.945 & 0.996 & -2.51 \\
\hline
\end{tabular}

$\mathrm{cmc}^{\mathrm{ex}}$ the experimentally obtained $\mathrm{cmc}$ values of investigated binary systems, each result is the average of three separate determinations.

$\mathrm{cmc}^{i d}$ the values for the critical micelle concentrations of ideal mixtures, calculated according to Clint's theory of ideal mixtures.

$x_{1}$ mole fractions of the more hydrophobic surfactant in the real mixed micelles, according to Rubingh.

$x^{i d}$ mole fractions of the more hydrophobic surfactant in the ideal mixed micelles, according to Motomura's model.

anionic heads are important source of destabilisation $[49,50]$. Hence, weak synergistic or antagonistic interactions between individual surfactants are frequently reported in anionic-anionic surfactant mixtures $[26,49,50]$. Therefore, weak synergism that has been noticed for anionic-anionic surfactant mixtures in this article is in agreement with previous studies.

The results presented in Table 7 show that TRX and SLES generate the weakest synergistic effect with D, while OD creates the strongest interaction with investigated co-surfactants. We assume that the hydrophobic regions of TRX and SLES are incorporated in the micellar core of investigated binary systems. The polar heads of SLES and TRX are probably located at the surface of the aggregates, interacting with the polar $\alpha$ side of the bile salts, as presented in Figure 3. for TRX-OD binary system. Consequently, the variation in synergism between investigated bile salts and selected co-surfactants can be attributed to the structural differences in the polar part of the steroid skeleton.
Table 6 Comparison of experimental with ideal parameters and interaction parameter $(\beta)$ of the SLES-bile salt mixtures

\begin{tabular}{|c|c|c|c|c|c|}
\hline \multirow[t]{2}{*}{$\alpha$ [SLES] } & $c m c^{e x}$ & $c m c^{i d}$ & \multirow[t]{2}{*}{$x_{1}$} & \multirow[t]{2}{*}{$x^{i d}$} & \multirow[t]{2}{*}{$\beta$} \\
\hline & \multicolumn{2}{|c|}{$(\mathrm{mM})$} & & & \\
\hline \multicolumn{6}{|c|}{ Sodium deoxycholate } \\
\hline 0 & 4.30 & - & - & - & - \\
\hline 0.1 & 2.857 & 3 & 0.384 & 0.374 & -0.22 \\
\hline 0.2 & 2.203 & 2.29 & 0.566 & 0.573 & -0.16 \\
\hline 0.3 & 1.845 & 1.86 & 0.694 & 0.697 & -0.03 \\
\hline 0.4 & 1.528 & 1.56 & 0.769 & 0.782 & -0.12 \\
\hline 0.5 & 1.322 & 1.35 & 0.830 & 0.843 & -0.16 \\
\hline \multicolumn{6}{|c|}{ Sodium cholate } \\
\hline 0 & 12.90 & & - & - & - \\
\hline 0.1 & 4.461 & 5.15 & 0.611 & 0.644 & -0.60 \\
\hline 0.2 & 2.864 & 3.21 & 0.746 & 0.802 & -0.64 \\
\hline 0.3 & 2.165 & 2.33 & 0.826 & 0.874 & -0.57 \\
\hline 0.4 & 1.730 & 1.83 & 0.873 & 0.915 & -0.57 \\
\hline 0.5 & 1.427 & 1.51 & 0.899 & 0.942 & -0.78 \\
\hline \multicolumn{6}{|c|}{ Sodium 7-oxodeoxycholate } \\
\hline 0 & 61.10 & & & & \\
\hline 0.1 & 6.511 & 7.14 & 0.832 & 0.893 & -0.78 \\
\hline 0.2 & 3.598 & 3.8 & 0.906 & 0.949 & -0.81 \\
\hline 0.3 & 2.493 & 2.59 & 0.938 & 0.97 & -0.87 \\
\hline 0.4 & 1.906 & 1.96 & 0.955 & 0.98 & -1.03 \\
\hline 0.5 & 1.549 & 1.58 & 0.969 & 0.987 & -0.94 \\
\hline
\end{tabular}

$\mathrm{cmc}^{e x}$ the experimentally obtained values, each result is the average of three separate determinations.

$\mathrm{cmc}^{i d}$ the values for the critical micelle concentrations of ideal mixtures, calculated according to Clint's theory of ideal mixtures.

$x_{1}$ mole fractions of the more hydrophobic surfactant in the real mixed micelles, according to Rubingh.

$x^{\text {id }}$ mole fractions of the more hydrophobic surfactant in the ideal mixed micelles, according to Motomura's model.

Obviously, the larger number of hydrophilic groups on $\alpha$ side of the steroid ring generates stronger synergism, since $C$ and OD have one polar group more than DC (7-hydroxyl and 7-oxo group, respectively).

Namely, it is possible that $\alpha$-hydroxyl groups on the steroid skeleton create hydrogen bonds with proton acceptor OE groups of the polar head of TRX and SLES. It is known that DC lacks 7-hydroxyl group, thus most likely forms fewer hydrogen bonds.

Stronger synergistic effect of OD in all investigated binary systems compared to the same surfactant systems with $\mathrm{C}$ is probably the consequence of the alteration in the spatial position of the oxygen atom at $\mathrm{C} 7$ of the steroid skeleton. It is known that $\alpha$-axial hydroxyl group at $\mathrm{C} 7$ position of the steroid skeleton forms a $90^{\circ}$ angle with the median plane of the steroid skeleton (MPSC). Oxidation of this group creates $\alpha$-equatorial oxo group which forms a $30^{\circ}$ angle with the median plane of the steroid skeleton (MPSC) as seen in Figure 4. Therefore, the oxygen atom of the $\mathrm{C} 7$ oxo group is moved for $60^{\circ}$ 
Table 7 Average $\beta$ interaction parameters $\left(\beta_{a v}\right)$ of analysed mixed binary systems

\begin{tabular}{ccc}
\hline Surfactant & TRX & SLES \\
& $\boldsymbol{\beta}_{\boldsymbol{a v}}$ & $\boldsymbol{\beta}_{\boldsymbol{a v}}$ \\
\hline $\mathrm{D}$ & -0.88 & -0.14 \\
$\mathrm{C}$ & -2.06 & -0.63 \\
$\mathrm{OD}$ & -2.52 & -0.89 \\
\hline
\end{tabular}

towards the MPSC i.e. towards the $\beta$ side of the steroid ring.

We assume that this spatial relation of the oxygen atom enables polar head (OE groups) of the co-surfactants to be oriented towards the $\alpha$-axial hydroxyl groups of the OD steroid skeleton, since hydrophilic region of the cosurfactants is probably located at the micellar surface.

This spatial orientation of TRX is probably achieved through the water molecules, as seen in Figure 3. We assume that similar positioning is attained with SLES molecules, through the dipole interactions between the OE group distal to sulfate ion and the 7-oxo group of OD. According to the results, TRX shows stronger synergism with investigated bile salts than SLES. Apart from difference in hydrophobic region, this variation can be explained due to the smaller number of $\mathrm{OE}$ groups in hydrophilic region of SLES, forming fewer attractive interactions with the polar part of selected bile salts. Additionally, negatively charged sulfate ion of SLES creates repulsive interactions with carboxylate anion of selected bile salts, decreasing stability of mixed micelles.

\section{Conclusion}

We have studied a set of three bile salts (sodium cholate, sodium deoxycholate or sodium 7-oxodeoxycholate)

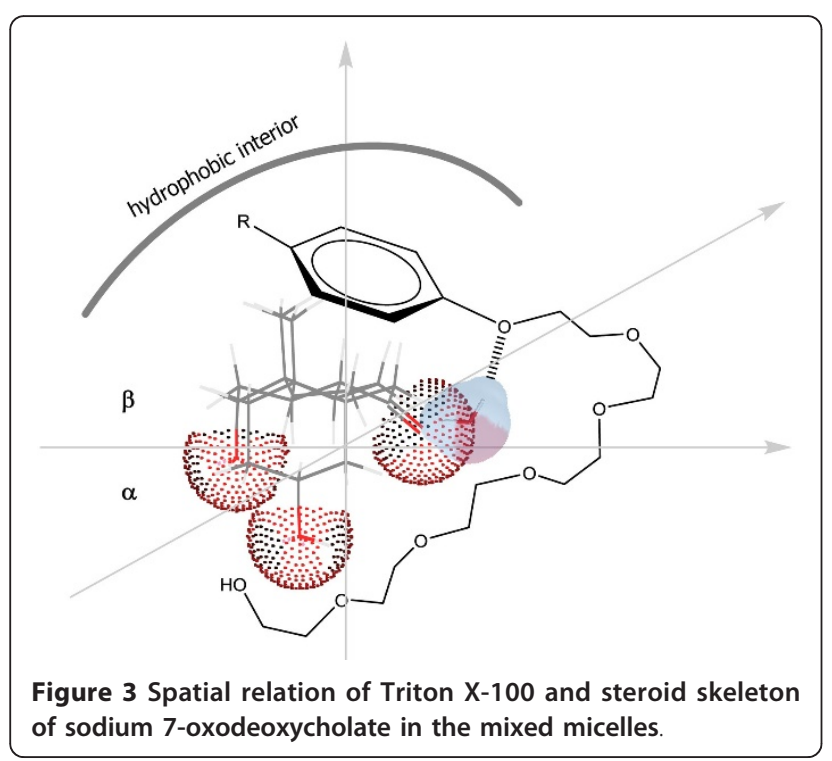

to interact with Triton X-100 or sodium lauryl ether sulfate, by conductometric and tensiometric method. The results of the study have been analysed using Clint's, Rubingh's, and Motomura's theories for mixed binary systems. The critical micelle concentration of the ideal mixed micelle, the mole fraction of the more hydrophobic surfactant in the ideal mixed micelle, the mole fraction of the more hydrophobic surfactant in the real mixed micelle, and the $\beta$ interaction parameter of the mixed micelles were calculated using experimental data.

Based on the calculated values of the $\beta$ parameter we have concluded that TRX and SLES generate the weakest interactions with $\mathrm{D}$, while $\mathrm{OD}$ creates the strongest interaction with investigated co-surfactants. Most probably, hydrogen bonds between $\alpha$-hydroxyl groups of steroid skeleton and proton acceptor OE groups of the polar head of selected co-surfactants are formed and contribute to the synergism. Since D lacks one polar group compared to $\mathrm{C}$ and $\mathrm{OD}$, it showed the weakest interactions.

We have also noticed that OD generates stronger synergism than $C$ in all investigated binary systems. This is probably due to attractive interactions between 7-oxo group of OD and the oxygen atom of the phenoxy ring of TRX through the water molecules. This effect favours attractive intermolecular hydrophilic interactions and formation of hydrogen bonds.

It is assumed that attractive interaction between 7keto group of OD and of the second OE group distal to sulfate group in SLES promotes formation of hydrogen bonds and stabilisation of the binary system.

\section{Experimental}

\subsection{Materials}

Sodium 7-oxodeoxycholate was synthetized by selective oxidation of the $7 \alpha$-hydroxy group of cholic acid, following the Tullar procedure [51]. All bile acids were transformed to the sodium salt by known procedure [3]. Cholic and deoxycholic acids were purchased from Sigma-Aldrich (Germany). The degree of purity is $>99 \%$. Triton X-100 was obtained from J.T. Baker (Holland). The degree of purity is $>99 \%$. Sodium lauryl ether sulfate having two oxyethylen groups was provided by Stepan Company, USA. Surfactant solutions were prepared by dissolving accurately weighed quantities of surfactants in requisite volumes of deionised water. Deionised water (conductivity $<1 \mu \mathrm{S} \mathrm{cm}{ }^{-1}$, at $25^{\circ} \mathrm{C}$ ) was used for all purposes.

\subsection{Methods}

\subsubsection{Critical micelle concentration determination}

The $c m c$ values of pure $\mathrm{C}, \mathrm{D}, \mathrm{OD}$ and SLES were acquired through conductivity and surface tension 


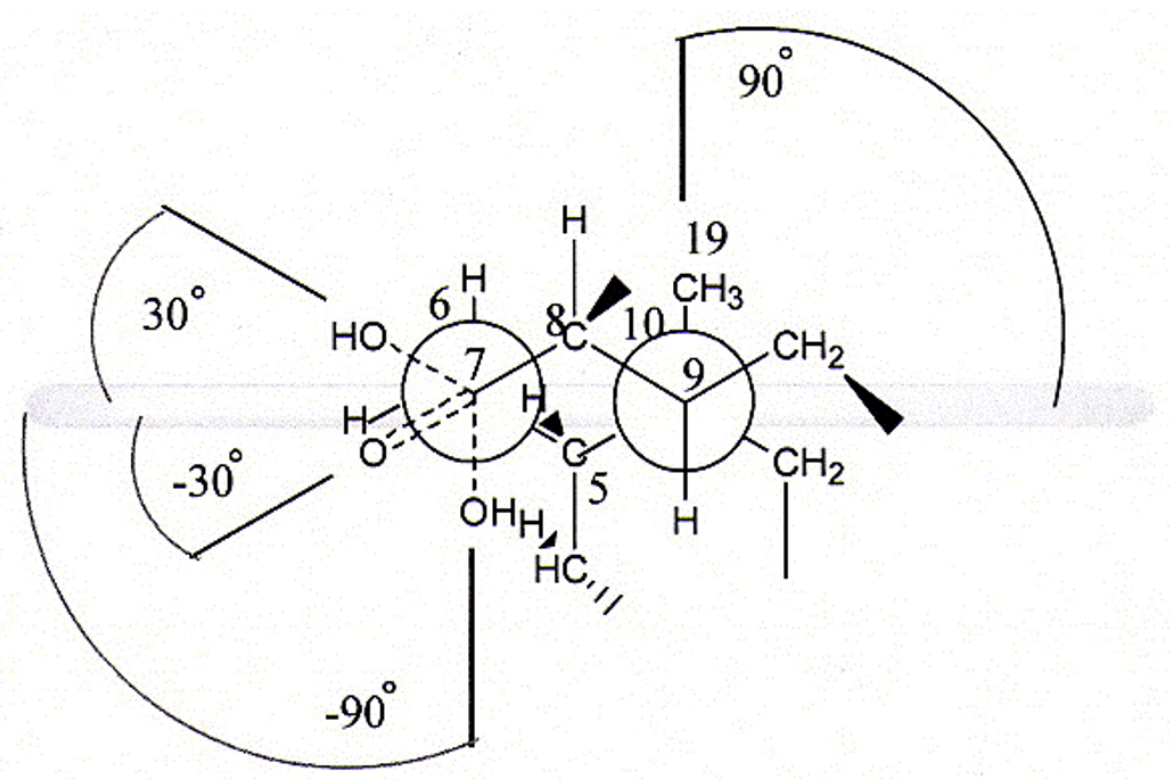

Figure 4 Spatial position modification of the oxygen atom at C7 (Newman projection of sodium 7-oxodeoxycholate).

measurements. The $c m c$ value of TRX was obtained through surface tension studies only, since this surfactant is nonionic amphiphile and does not have influence on electric conductance. The critical micelle concentrations of all investigated binary mixtures (D-TRX, C-TRX, ODTRX, D-SLES, C-SLES, and OD-SLES) were determined by means of conductometry and tensiometry.

\subsubsection{Conductivity measurements}

Conductivity measurements were carried out on aqueous solutions of pure C, D, OD and SLES. Aqueous solutions of mixtures of $\mathrm{C}, \mathrm{D}$ or $\mathrm{OD}$ and one of two Cosurfactants (TRX or SLES) were also analysed measuring conductivities. The $c m c$ values of the binary systems were obtained for the different molar ratios of the components. Conductivities were measured by gradual dilution of surfactant solutions with the deionised water. The data were acquired using Consort C 860 conductometer. The cell containing solutions was immersed in a water bath, controlling the temperature variation at \pm $0.1^{\circ} \mathrm{C}$. The temperature was kept constant at $25^{\circ} \mathrm{C}$. Trials were repeated three times for reproducibility. The cmc determination error did not exceed 3\%.

\subsubsection{Surface tension measurements}

Surface tension measurements were carried out on aqueous solution of TRX in order to determine its individual $c m c$. Surface tension measurements were carried out on a Sigma 703D tensiometer (Finland) using a du Nouy ring method. All measurements were repeated three times. In all measurements temperature was kept constant at $25 \pm 0.1^{\circ} \mathrm{C}$. The $\mathrm{cmc}$ determination error did not exceed $3 \%$.

\section{Acknowledgements}

This work was financially supported by the Provincial Secretariat for Science and Technological Development, AP Vojvodina, Republic of Serbia, Grant No.114-4512113/2011-01

\section{Authors' contributions}

All three authors initiated and designed the study. All calculations are carried out by DMĆ, MMP and VSK. Authors contributed equally to data analyses and to finalizing the manuscript. All authors have read and approved the final version of the manuscript.

\section{Competing interests}

The authors declare that they have no competing interests.

Received: 5 November 2011 Accepted: 29 December 2011 Published: 29 December 2011

\section{References}

1. Armstrong MJ, Carey MC: The hydrophobic-hydrophilic balance of bile salts. Inverse correlation between reverse-phase high performance liquid chromatographic mobilities and micellar cholesterol-solubilizing capacities. J Lipid Res 1982, 23:70-80.

2. Gardiel P, Hildebrand A, Knauf K, Blume A: Membranolytic Activity of Bile Salts: Influence of Biological Membrane Properties and Composition. Molecules 2007, 12:2292-2326.

3. Roda A, Hofmann AF, Mysels KJ: The influence of bile salts structure on selfassociation in aqueous solution. J Biol Chem 1983, 258:6362-6370

4. Madenci D, Egelhaaf SU: Self-assembly in aqueous bile salt solutions. Curr Opin Colloid Interface Sci 2010, 15:109-115.

5. Haslewood GAD: Bile salt evolution. J Lipid Res 1967, 8:535-550

6. Small DM: The physical chemistry of cholanic acids. In The Bile Acids: Chemistry, Physiology and Metabolism. Volume 1. Edited by: Nair PP, Kritchevsky D. Plenum Press New York; 1971, Chapter 8.

7. Poša M, Guzsvany V, Csanadi J: Determination of critical micellar concentration of two monoketo derivatives of cholic acid. Colloids Surf Biointerface 2009, 74:84-90. 
8. Bowe CL, Mokhtarzadeh L, nVenkatesen P, Babu S, Axelrod H, Sofia MJ, Kakarla R, Chan TY, Kim JS, Lee HJ, Amidon GL, Choe SY, Walker S, Kahne D: Design of compounds that increase the absorption of polar molecules. Proc Natl Acad Sci USA 1997, 94:12218-12223.

9. Gordon GS, Moses AC, Silver RD, Flier JR, Carey MC: Nasal absorption of insulin: enhancement by hydrophobic bile salts. Proc Natl Acad Sci USA 1985, 82:7419-7423.

10. Camile W: The Practice of Medicinal Chemistry Oxford: Academic Press; 2003.

11. De Castro B, Gameiro P, Guimaraes C, Lima JLFC, Reis S: Study of partition of nitrazepam in bile salt micelles and the role of lecithin. $J$ Pharm Biomed Anal 2001, 24:595-602.

12. Wiedmann TS, Kamel L: Examination of the solubilization of drugs by bile salt micelles. J Pharm Sci 2002, 91:1743-1764.

13. Ninomiya R, Matsuoka K, Moroi Y: Micelle formation of sodium chenodeoxycholate and solubilization into the micelles: comparison with other unconjugated bile salts. Biochim Biophys Acta 2003, 1634:116-125.

14. Al-Ahmadi MDA, Naqvi AZ, Akram M: Conductometric study of antidepressant drug-cationic surfactant mixed micelles in aqueous solution. Colloids Surf Biointerface 2008, 64:65-69.

15. Al-Ahmadi MDA, Naqvi AZ, Akram M: Micellar Properties of a Phenothiazine Drug in Presence of Additives. Colloid J Russia 2009, 71:498-502.

16. Poša M, Tepavčević $V$ : Mixed micelles of 7,12-dioxolithocholic acid and selected hydrophobic bile acids: interaction parameter, partition coefficient of nitrazepam and mixed micelles haemolytic potential. Colloids Surf Biointerface 2011, 86:285-291.

17. Bhattacharjee J, Verma G, Aswal VK, Date AA, Nagarsenker MS, Hassan PA: Tween 80-sodium deoxycholate mixed micelles: structural characterization and application in doxorubicin delivery. J Phys Chem B 2010, 114:16414-16421.

18. Yu JN, Zhu Y, Wang L, Peng M, Tong SS, Cao X, Qiu H, Xu XM: Enhancement of oral bioavailability of the poorly water-soluble drug silybin by sodium cholate/phospholipid-mixed micelles. Acta Pharmacol $\sin 2010,31: 759-764$

19. Sznitowska M, Klunder M, Placzek M: Paclitaxel solubility in aqueous dispersions and mixed micellar solutions of lecithin. Chem Pharm Bull 2008, 56:70-74

20. Liu J, Gong T, Wang C, Zhong Z, Zhang Z: Solid lipid nanoparticles loaded with insulin by sodium cholate-phosphatidylcholine-based mixed micelles: preparation and characterization. Int J Pharm 2007, 34:153-162.

21. Guo J, Wu T, Ping Q, Chen Y, Shen J, Jiang G: Solubilization and pharmacokinetic behaviors of sodium cholate/lecithin-mixed micelles containing cyclosporine A. Drug Deliv 2005, 12:35-39.

22. De Castro B, Gameiro P, Guimarães C, Lima JL, Reis S: Partition coefficients of beta-blockers in bile salt/lecithin micelles as a tool to assess the role of mixed micelles in gastrointestinal absorption. Biophys Chem 2001, 90:31-43.

23. Hammad MA, Müller BW: Increasing drug solubility by means of bile saltphosphatidylcholine-based mixed micelles. Eur J Pharm Biopharm 1998, 46:361-367.

24. Reis S, Moutinho CG, Pereira E, de Castro B, Gameiro P, Lima JL: Betablockers and benzodiazepines location in SDS and bile salt micellar systems. An ESR study. J Pharm Biomed Anal 2007, 45:62-69.

25. Wiedmer SK, Riekkola ML: Mixed Micelles of Sodium Dodecyl Sulfate and Sodium Cholate: Micellar Electrokinetic Capillary Chromatography and Nuclear Magnetic Resonance Spectroscopy. Anal Chem 1997, 69:1577-1584.

26. Haque ME, Das AR, Rakshit AK, Moulik SP: Properties of Mixed Micelles of Binary Surfactant Combinations. Langmuir 1996, 12:4084-4089.

27. Wiedmer SK, Nydeacuten M, Soumlderman O, Riekkola ML: Mixed micelles of SDS and sodium cholate. A nuclear magnetic resinance diffusion and relaxation study. J Dispersion Sci Technol 2000, 21:209-227.

28. Haque ME, Das AR, Moulik SP: Behaviors of sodium deoxycholate (NaDC) and polyoxyethylene tert-octylphenyl ether (Triton X-100) at the air/ water interface and in the bulk. J Phys Chem 1995, 99:14032-14038.

29. Haque ME, Das AR, Moulik SP: Mixed micelles of sodium deoxycholate and polyoxyethylene sorbitan monooleate (Tween 80). J Colloid Interface Sci 1999, 217:1-7.

30. Clint JH: Micellization of mixed nonionic surface active agents. J Chem Soc Faraday Trans / 1975, 71:1327-1334.
31. Motomura K, Aratono M: In Mixed Surfactant Systems. Edited by: Ogino K, Abe M. Marcel Dekker: New York; 1993:99-144.

32. Rubingh DN: In Solution Chemistry of Surfactants. Edited by: Mittal KL. Plenum Press: New York; 1979:337-354.

33. Nakamura $\mathrm{H}$, Sano A, Matsuura K: Determination of critical micelle concentration of anionic surfactants by capillary electrophoresis using 2naphthalenemethanol as a marker for micelle formation. Anal Sci 1998, 14:379-382.

34. Hait SK, Moulik SP: Determination of critical micelle concentration (CMC) of nonionic surfactants by donor-acceptor interaction with lodine and correlation of CMC with hydrophile-lipophile balance and other parameters of the surfactants. J Surfactants Deterg 2001, 4:303-309.

35. Aoudia M, Al-Maamari T, Al-Salmi F: Intramolecular and intermolecular ion- dipole interactions in sodium lauryl ether sulfates (SLES) selfaggregation and mixed micellization with Triton X-100. Colloids and Surfaces A: Physicochem Eng Aspects 2009, 335:55-61.

36. Otto M: Chemometrics: Statistics and Computer Application in Analytical Chemistry Weinheim: WILEY-VCH; 2007.

37. Philips JN: Energetics of micelle formation. Trans Faraday Soc 1955, 51:561-569.

38. Mosquera V, Garcia M, Varela NM: Association colloids: CMC a property to calculate. In Handbook of Surfaces and Interfaces of Materials. Edited by: Nalwa HS. New York: Academic Press; 2001:405-426.

39. Munoz M, Rodriguez A, Graciani MM, Moya ML: Conductometric, surface tension, and kinetic studies in mixed SDS-Tween 20 and SDS-SB3-12 micellar solutions. Langmuir 2004, 20:10858-10867.

40. Garcia-Mateos I, Velazquez MM, Rodriguez LJ: Critical micelle concentration determination in binary mixtures of lonic surfactants by deconvolution of conductivity/concentration curves. Langmuir 1990, 6:1078-1083.

41. Grillo I, Penfold J: Self-assembly of mixed anionic and nonionic surfactants in aqueous solution. Langmuir 2011, 27:7453-7463.

42. Jiang $Y$, Chen H Mao S, Luo P, Du Y, Liu M: Dynamics of mixed surfactants in aqueous solutions. J Phys Chem B 2011, 115:1986-1990.

43. Akbar JR, Deubry R, Marangoni DG, Wettig SD: Interactions between gemini and nonionic pharmaceutical surfactants. Can J Chem 2010, 88:1262-1270.

44. Cui $X$, Jiang $Y$, Yang $C$, Lu X, Chen H, Mao S, Liu M, Yuan H, Luo P, Du Y: Mechanism of the mixed surfactant micelle formation. J Phys Chem $B$ 2010, 114:7808-7816

45. Muherei MA, Junin R: Investigating synergism in critical micelle concentration of anionic-nonionic surfactant mixtures: surface versus interfacial tension techniques. Asian J App/ Sci 2009, 2:115-127.

46. Patel T, Ghosh G, Aswal V, Bahadur P: Micellization of sodium dodecyl sulfate and polyoxyethylene dodecyl ethers in solution. Colloid Polym Sci 2009, 287:1175-1181.

47. Azzam EMS: The synergism effect between sodium dodecylbenzene sulfonate and a block copolymer in aqueous solution. J Surfactants Deterg 2007, 10:13-17.

48. Owoyomi O, Jide I, Akanni MS, Soriyan OO, Morakinyo MK: Interactions between sodium dodecylsulphate and Triton X-100: Molecular properties and kinetics investigation. J App/ Sci 2005, 5:729-734

49. Rodriguez-Pulido A, Casado A, Munoz-Ubeda M, Junquera E, Aicart E: Experimental and theoretical approach to the sodium decanoatedodecanoate mixed surfactant system in aqueous solution. Langmuir 2010, 26:9378-9385.

50. Vora S, George A, Desai H, Bahadur P: Mixed micelles of some anionicanionic, cationic-cationic and ionic-nonionic surfactants in aqueous media. J Surf Deterg 1999, 2:213-221.

51. Tullar BF: Hemi-diethylene glycol adduct of 3, 12-dihydroxy-7ketocholanic acid and preparation thereof. U.S. Patent 2,549,947; 1951.

doi:10.1186/1752-153X-5-89

Cite this article as: Ćirin et al.: Interactions between selected bile salts and Triton X-100 or sodium lauryl ether sulfate. Chemistry Central Journal $20115: 89$. 